\title{
Emergence of antibiotic-resistant bacterial strains, methicillin-resistant Staphylococcus aureus, extended spectrum beta lactamases, and multi-drug resistance is a problem similar to global warming
}

\author{
Pranab Kumar Bhattacharya ${ }^{[1]}$
}

[1]. Department of Pathology, Murshidabad District Medical College, Berhampore, Murshidabad, West Bengal, India.

Dear Editor,

I recently read the article by Porto et al. entitled Active surveillance to determine the impact of methicillin resistance on mortality in patients with bacteremia and influences of the use of antibiotics on the development of MRSA infection, published in Revista da Sociedade Brasileira de Medicina Tropical/Journal of the Brazilian Society of Tropical Medicine ${ }^{1}$.

Methicillin-resistant Staphylococcus aureus (MRSA) is a major pathogen worldwide in modern healthcare-associated infections. Within 1 year of introducing methicillin in clinical practice, MRSA strains were first detected in 1961 in Britain, followed by Denmark in 1963, and subsequently other parts of the world. MRSA is associated with increased morbidity and mortality from sepsis and rapidly develops multi-drug resistance (MDR). In a hospital-based study at Kolkata metro City by the author, MRSA showed in vitro drug resistance to co-trimoxazole (33\%), erythromycin $(75 \%)$, penicillin $(22 \%)$, oxacillin $(11 \%)$, ciprofloxacin $(84 \%)$, mupirocin $(8 \%)$, rifampicin $(17 \%)$, and methicillin (85\%); and MRSA also showed in vitro sensitivity to clindamycin $(90 \%)$, cefotaxin $(99 \%)$, vancomycin $(100 \%)$ and linezolid $(100 \%)$. Linezolid and vancomycin have been clinically used as reliable last resort drugs for MRSA treatment. Since 1997, vancomycin-intermediate and -resistant $S$. aureus and linezolid-resistant strains of $S$. aureus have been reported $^{2,3}$. Data on linezolid resistance from Asian countries, especially from the Indian subcontinent, are extremely rare, and Chakraborty et al. ${ }^{4}$ found only a single case of linezolidresistant MRSA in a 33-year-old male patient with non-healing ulcers in a tertiary care medical college using polymerase chain reaction (PCR) to detect: I) mutations in the domain $\mathrm{V}$ region of one or more of the five copies of the $23 \mathrm{~S}$ recombinant deoxyribonucleic acid (rDNA) gene; II) acquisition of the

\footnotetext{
Address to: Dr. Pranab Kumar Bhattacharya. Depto of Pathology/District Medical College. Station Road 742101 Berhampore, Murshidabad, West Bengal, India.

Phone: 9192 3151-0435

e-mail: profpkb@yahoo.co.in

Received 11 June 2014

Accepted 26 November 2014
}

plasmid-mediated ribosomal methyl transferase Cfr gene, and III) deletion/mutation in the ribosomal protein L3 of peptidyl transferase center ${ }^{5}$.

The prevalence of MRSA has increased in most private health care institutions, including Kolkata City of West Bengal, India, where it increased from $6.9 \%$ in 1988 , to $54 \%$ in 2003 , to $63 \%$ in 2013 , varying from $8 \%$ to $71 \%$ across India 6 . Resistance is due to an altered penicillin binding protein encoded by the mecA gene. MRSA can be confirmed using multiplex PCR-based detection of the mecA2 gene using the ATA3 'mecA1 forward (5 GTAGAAATGACTGAACGTCCG) and reverse (5 CCATTCCACATTGTTTC) primers, as well as the oxacillin disk diffusion (ODD) and cefoxitin disk diffusion (CDD) tests. Although considered the gold standard for MRSA diagnosis, PCR for the mecA gene is costly and requires a skilled technician; thus, many tertiary care hospitals and even medical colleges or private centers in West Bengal cannot afford to routinely perform PCR. ODD has a sensitivity of $97 \%$, specificity of $95 \%$, and an effectiveness of $97 \%$; and CDD has sensitivity, specificity, and effectiveness of $100 \%$ for the diagnosis of MRSA infection when compared with PCR for mecA. In Kolkata, common causative organisms of hospital-acquired infections are Staphylococcus aureus, Streptococcus viridans, aerobic gram-negative bacilli such as Pseudomonas aeruginosa, Escherichia coli, and other MDR gram-negative organisms, including Acinobacter, Klebsiella, E. coli, Salmonella, and extended spectrum beta lactamase (ESBL) enterobacteriaceae (E. coli and Klebsiella; prevalence,62-100\%), and $S$. aureus, responsible for various mild to serious infections in multiple hospital units, leading to a higher median total hospital cost, longer hospital stay after infection (mean 22.9 days 6 ), fatal sepsis, and death (mean $84 \% \%^{5}$ ). MRSA and methicillin-sensitive $S$. aureus strains with quinolone resistance have also been reported from an eye care hospital in Bhubaneswar, India ${ }^{7,8}$.

Community-associated MRSA(CAMRSA) currently comprises $40-60 \%$ of all nosocomial $S$. aureus infections and cause sepsis at many centers worldwide, is one of the most prevalent significant pathogens, and causes a variety of infections from mild skin and soft tissue infections and 
necrotizing fasciitis to fatal sepsis. In one of our studies, when 336 individuals in the community were randomly screened from their deeper nasal cavity for persistence of $S$. auerus, the first 136 subjects showed no MRSA, whereas in the next 200 subjects, only $11 \%$ showed MRSA. In another study from Wardha, India, of 280 CAMRSA samples, $51.8 \%$ had the $m e c A$ gene $^{5}$. CAMRSA colonizes the anterior nares, and nasal carriage of $S$. aureus acts as an endogenous reservoir for clinical infections in colonized individuals or as a source of crosscolonization for community-based infections. Nasal S. auerus colonization is an important risk factor for life-threatening infections of the carrier. In our CAMRSA study, the overall incidence of $S$. aureus was $12-28 \%$, even in healthy subjects: a higher prevalence may occur in overcrowded and slum areas.

MRSA results in surgical site infection, non-healing ulcer, necrotizing fasciitis, pneumonia, urinary tract infection, and central venous catheter-associated infections. Due to use of previous third-generation antibiotics in critical care units, coagulase-negative staphylococci cause major infections, followed by Klebsiella pneumoniae, Pseudomonas aeruginosa, and non-albicans Candida -producing bio films in urinary catheters; nasogastric, parenteral nutrition, and tracheostomy tubes; and mechanical ventilators. Risk factors and mortality rate are always higher with MRSA infection. Comorbid conditions that act as risk factors include cancer, heart disease, nephropathy, diabetes mellitus, chronic obstructive pulmonary disease, polytrauma, dyslipidemia, human immunodeficiency virus, cystic fibrosis, hepatitis, pulmonary emphysema, dialysis, and tobacco and marijuana smoking.

In 210 samples the neonatal intensive care unit of a tertiary care public hospital in Kolkata, the most common isolated bacteria causing sepsis (all with a high C-reactive protein value) included Klebsiella (42.3\%), MRSA (32.1\%), and coagulase-negative staphylococci (11.1\%). Of the 44 MRSA infection cases, 2-15\% showed resistance to amikacin, gentamycin, cloxacillin, ofloxacin, amoxicillin/clavulanic acid, levofloxacin, cefotaxine, and co-trimoxazole, in addition to vancomycin $(100 \%)^{4}$.

Today, the persistent, indiscriminate, and inappropriate use of antibiotics and the increasing specter of antibiotic resistance are an emerging critical situation for healthcare. This situation needs immediate action with current anti-infective therapies in West Bengal. MDR organisms will continue to increase unless clinicians in most care hospitals and private practices improve the rational use of antibiotics. The matter is rendered increasingly complicated due to the presence of ESBL and carbapenamase-producing organisms due to the blinded use of third and fourth-generation antibiotics. The potential for misuse and abuse of antibiotics was recognized shortly after their introduction into West Bengal; this may have been further escalated by failure to obtain appropriate culture and in vitro sensitivity reports for lower respiratory and urinary tract infections and pus. Meropenem or tazobactam is routinely used as the choice first-line antibiotic in most private care hospitals and nursing homes and for some doctor's prescriptions; this is due to kickbacks in the form of monetary commissions from pharmacy houses. We need urgent implementation of an effective antibiotic policy, and the basis of that policy rests on generating microbiological data and prescription audits through electronic prescriptions to be introduced to all public and private health care institutes(where approximately $60 \%$ of antimicrobial use is inappropriate) or at any geographical location where drug resistance has developed.

Antibiotic resistance is no less a problem than global warming. Moreover, the overuse of antibiotics causes the emergence of bacterial resistance and increases healthcare costs and sepsis-related deaths. We propose the development of a new global organization to lead the battle against antibioticresistant pathogens and call for this organization to be modeled on the organization created to combat global warming, the Intergovernmental Panel on Climate Change. This organization would be comprised of specialists in clinical and pathological medicine and include epidemiologists, microbiologists, pharmacologists, health economists, and international lawyers.

\section{ACKNOWLEDGMENTS}

The author acknowledges the following individuals for their help: Upasana Bhattacharya, Rupak Bhattacharya, Ritwik Bhattacharya, Rupsa Bhattacharya, Hindole Mukherjee, Dalia Mukherjee, Oindrila Mukherjee, Ayishi Mukherjee, Arya Sen and Prof. Pratip Kundu.

\section{REFERENCES}

1. Porto JP, Santos RO, Gontijo Filho P, Ribas RM. Active surveillance to determine the impact of methicillin resistance on mortality in patients with bacteremia and influences of the use of antibiotics on the development of MRSA infection. Rev Soc Bras Med Trop 2013; 46:713-718.

2. Hiramtsu K, Hanaki H, Ino T, Yabuta K, Oguri T, Tenover FC. Methicillin-resistant Staphylococcus aureus clinical strain with reduced vancomycin susceptibility. J Antimicrob Chemother 1997; 40:135-136.

3. Centers for Disease Control and Prevention (CDC). Staphylococcus aureus resistant to vancomyci--United States, 2002. MMWR Morb Mortal Wkly Rep 2002; 51:565-567.

4. Chakraborty B, Chakraborty B, Dopthap YP, Banerjee D. LRSA suspect, taken to molecular court, PCR trialled, proven guilty. Ann Trop Med Public Health 2013; 6:653-657.

5. Mallick S, Kumar, Basak S. Accurate detection of methicillinresistant Staphylococcus aureus in day to day practice: a great help to clinicians. J Indian Med Assoc 2011; 109:892-895.

6. Bhattacharya S. Is screening for antibiotic-resistant bacteria justified in the Indian context? Indian J Med Microbiol 2011; 29:213-217.

7. Choudhury R, PandaS, SinghDV. Emergence and dissemination of antibiotic resistance: a global problem. Indian J Med Microbiol 2012; 30:384-390.

8. Choudhury R, PandaS, Sharma S, Singh DV. Staphylococcal infection, antibiotic resistance, and theraputics. In: Pana M, editor. Antibiotic resistant bacteria- a continuous challenge in the new millennium. Chapter 10. In Tech; 2012. p. 247-272. 